\title{
Factores de uso de los medios de pago móviles en millennials y centennials
}

\author{
https://doi.org/10.22395//seec.v22n53a4 \\ María Camila Bermeo-Giraldo* \\ Alejandro Valencia-Arias** \\ Bianeth Duque García ${ }^{* * * *}$ \\ Luis Fernando Garcés-Giraldo* \\ Teresa Luna-Ramírez $z^{* * * * *}$
}

Recibido: 12 de diciembre de 2019 • Aprobado: 26 de diciembre de 2019

\section{RESUMEN}

Las tecnologías de información implementadas en los sistemas de pago han incrementado la oferta de servicios mediante medios móviles, con una adopción aún limitada. Por ello, la relevancia de investigar sobre la utilización de los medios de pago virtuales a través de la identificación de los factores que inciden en la adopción de la banca móvil por jóvenes millennials y centennials de Medellín, mediante el análisis factorial exploratorio y análisis factorial confirmatorio de las variables: actitud de uso, conciencia percibida, credibilidad percibida, confianza percibida, intención de uso y experiencia de uso. La investigación empleó un análisis factorial exploratorio con un enfoque cuantitativo y de alcance exploratorio-descriptivo. Se concluye que las variables más relevantes en la decisión de usar pagos móviles son la experiencia en uso y la conciencia percibida.

\section{PALABRAS CLAVE}

Banca móvil; centennials; factores de utilización; millennials; modelo; tecnología bancaria.

\section{CLASIFICACIÓN JEL}

G21, O16, D14, G40

\section{CONTENIDO}

Introducción; 1. Millennials y centennials; 2. Sistema de pagos móviles; 3. Factores y estructuración del modelo; 4. Metodología; 5. Resultados; 6. Discusión; Conclusiones; Bibliografía.

\footnotetext{
El presente artículo es resultado del proyecto de investigación realizado con el Grupo de Investigación Interconexión Global y Finanzas, clasificado en Categoría C de Colciencias y titulado Percepción de la calidad del servicio en la banca móvil en la ciudad de Medellín, ejecutado en el periodo 2018-2019. La Institución Universitaria Escolme subvencionó su desarrollo.

. Ingeniera industrial de la Universidad Nacional de Colombia, sede Medellín, Colombia. Estudiante de Maestría en Ingeniería Administrativa en la Universidad Nacional de Colombia, sede Medellín, Colombia. Docente investigadora adscrita a la Institución Universitaria Escolme, Medellín, Colombia. Correo electrónico: cies2@escolme.edu.co. Orcid: https://orcid. org/0000-0001-6501-513X

... Ingeniero administrador y doctor en Ingeniería, Industria y Organizaciones, Universidad Nacional de Colombia, Medellín, Colombia. Docente investigador, Corporación Universitaria Americana, Medellín, Colombia. Investigador del Grupo de Investigación Engineeri@. Correo electrónico: javalenciar@gmail.com. Orcid: https://orcid.org/0000-0001-9434-6923

.... Tecnóloga en Gestión de Finanzas, Institución Universitaria Escolme, Medellín, Colombia. Correo electrónico: baduqueg@ escolme.edu.co. Orcid: https://orcid.org/0000-0001-8663-2495

..... Ingeniero sanitario, Universidad de Antioquia, Medellín, Colombia. Especialista en Ingeniería Ambiental, Universidad Pontificia Bolivariana, Medellín, Colombia. Magíster en Ingeniería Ambiental, Universidad de Antioquia, Medellín, Colombia. Doctor en Ciencias de la Atlantic International University, doctor en Filosofía, Pontificia Universidad Bolivariana, Medellín, Colombia. Vicerrector de Investigaciones, Corporación Universitaria Americana, Medellín Colombia. Investigador del Grupo de Investigación Aglaia. Correo electrónico: lgarces@americana.edu.co. Orcid: https://orcid.org/0000-0003-3286-8704

...... Ingeniera de sistemas, Universidad Cooperativa de Colombia, Medellín, Colombia. Especialista en Gerencia de la Universidad de San Buenaventura, Medellín, Colombia. Magíster en Educación, Atlantic International University. Decana de la Facultad de Ingeniería y Ciencias Ambientales, Fundación Universitaria Católica del Norte, Medellín, Colombia. Correo electrónico: tlunar@ucn.edu.co. Orcid: https://orcid.org/0000-0002-539-9909
} 


\title{
Factors of Use of Mobile Payment Means in Millennials and Centennials
}

\begin{abstract}
The information technologies implemented in the payment systems have increased the offer of services through mobile means, with a still limited adoption. Therefore, it became relevant to investigate the use of virtual payment methods through the identification of factors that affect the adoption of mobile banking in young millennials and centennials in Medellin, through exploratory factor analysis and factor analysis confirmation of the variables: attitude towards use, perceived awareness, perceived credibility, perceived trust, intention to use and experience of use. The research used exploratory factor analysis, with a quantitative and exploratory-descriptive approach. It is concluded that the most relevant variables in the decision to use mobile payment methods are use experience and perceived awareness.
\end{abstract}

KEYWORDS:

Mobile banking; banking technology; utilization factors; model; millennials; centennials.

JEL CLASSIFICATION

G21, O16, D14, G40

\section{CONTENTS}

Introduction; 1. Millennials and centennials; 2. Mobile payment system; 3. Factors and structuring of the model; 4. Methodology; 5 . Results; 6 . Discussion; Conclusions; Bibliography.

\section{Fatores de uso dos meios de pagamento móveis em millennials e centennials}

\section{RESUMO}

As tecnologias da informação do âmbito dos sistemas de pagamento têm aumentado a oferta de serviços mediante meios móveis, com uma adesão ainda limitada. Por isso, a relevância de pesquisar sobre a utilização dos meios de pagamento virtuais a partir da identificação dos fatores que incidem na adoção dessa modalidade por jovens millennials e centennials de Medellín, Colômbia, mediante a análise fatorial exploratória e a análise fatorial confirmatória das variáveis: atitudes de uso, consciência percebida, credibilidade percebida, confiança percebida, intenção de uso e experiência de uso. Esta pesquisa utilizou uma análise fatorial exploratória com uma abordagem quantitativa e de alcance exploratório-descritivo. Conclui-se que as variáveis mais relevantes na decisão de usar formas de pagamento com a tecnologia mobile payment são a experiência em uso e a consciência percebida.

\section{PALAVRAS-CHAVE}

Serviço bancário móvel; centennials; fatores de utilização; millennials; modelo; tecnologia bancária

\section{CLASSIFICAÇÃO JEL}

G21, O16, D14, G40

\section{CONTEÚDO}

Introdução; 1. Millennials e centennials; 2. Sistema mobile payment; 3. Fatores e estruturação do modelo; 4. Metodologia; 5. Resultados; 6. Discussão; Conclusões; Bibliografia. 


\section{INTRODUCCIÓN}

De acuerdo con Nie y Amarayoun (2018) y Yang y Lin (2018), con la implementación mundial de las tecnologías de la información, los medios virtuales se han convertido en parte de la vida cotidiana, al reemplazar las transacciones comerciales tradicionales. Además, para Acevedo-Prins y Jiménez-Gómez (2015), potencializar el uso de las tecnologías y mejorar la infraestructura tecnológica en el sector empresarial son factores que aportan a la competitividad de un país. En este contexto, para Aghili y Mala (2019), emplear el comercio electrónico (e-commerce), mediante el uso de dispositivos móviles y redes inalámbricas es conocido bajo el término m-commerce. Por su parte, Alalwan et al. (2018), indican que la principal ventaja del $m$-commerce es que permite acceder a cualquier producto de manera inmediata en cualquier momento, por lo que las personas disfrutan de servicios más rápidos en el sitio que quieran, incluso el mismo día. Durante los últimos años, la tecnología y el internet han sido utilizados ampliamente y han recibido cada vez más atención, lo que podría ser un factor esencial para el crecimiento de la economía mundial y conducir a la mejora de la calidad de vida de los seres humanos, como lo mencionan Banu, Ganagavalli y Ramsundar (2018) y Thammarat y Kurutach (2019), ya que se ha observado que a medida que la cantidad de dispositivos móviles crece, más minoristas en línea incorporan el comercio móvil como estrategia comercial.

Además, como lo expresan Medina (2011), Moreno-Agudelo y Valencia-Arias (2017) y Li et al. (2018), a pesar de los servicios ofrecidos aún hay dificultades en la adopción de los medios móviles para realizar pagos, dado que de acuerdo con la compañía Groupe Spéciale Mobile Association (GSMA) y con los autores Yeh et al., (2018) y Khalilzadeh, Ozturk, y Bilgihan (2017), el tema de seguridad es el aspecto más importante en la decisión de utilizar cualquier dispositivo para realizar pagos. Así, a medida que el uso de los dispositivos móviles se hace más popular entre los jóvenes, la seguridad de la información suministrada se vuelve indispensable para aumentar la confianza de los clientes. De allí, Trojanowski y Kułak (2018) y Ahamad y Pathan (2019), consideran que el tipo de aplicaciones, la complejidad, las transacciones y la identificación del usuario son preocupaciones clave del ecosistema del uso de estos medios, puesto que, hoy en día el uso de estos no garantiza la comunicación de extremo a extremo, la privacidad de la información y el anonimato del cliente. Sin embargo, Shaw y Sergueeva (2019) mencionan que los usuarios, al compartir información personal, reciben un servicio más rápido y personalizado. Debido al riesgo de pérdida de privacidad, los consumidores deberían equilibrar sus preocupaciones de seguridad con el valor percibido del comercio móvil mejorado. Otros problemas encontrados por Li et al., (2018) comprenden la satisfacción de las necesidades diarias de los consumidores basadas en la autenticación del usuario que 
inicia sesión en el sistema, la ayuda o soporte técnico, el respaldo de la transacción fuera de línea y el mejoramiento en la validez del pago.

En la literatura se han identificado factores que influyen en el uso de la tecnología móvil para realizar pagos, por ejemplo, Medrano y Díez (2010) analizaron el desconocimiento de los servicios ofrecidos en línea como una de las razones del bajo acceso a estos, ocasionado por barreras de oferta. Otro de los aspectos que influyen en el bajo uso de esta tecnología es la autoexclusión, definida como la preferencia por contar con liquidez o la percepción de inseguridad y desconfianza en el sistema financiero. Además, Nie y Amarayoun (2018) y Trojanowski y Kułak (2018) afirman que la facilidad de uso percibida también influye en la intención de utilizar dichos canales de pago.

Adicionalmente, Arango, Zarate-Solano y Suárez-Ariza (2017) afirman que aunque en Colombia el 70 \% de los pagos se efectúa de forma electrónica, solo el 9,8 \% corresponde al uso de dispositivos electrónicos y en línea. Al revisar los pagos realizados por personas naturales, los colombianos realizan un 3,8 \% del total de estas transacciones, es decir que en promedio, en el país un individuo hace uso de los teléfonos móviles 12 veces al año, mientras que en otros países, como Chile, sus habitantes hacen dicho uso 46 veces; en Brasil 135 y en Estados Unidos 347.

Para Posada y White (2016), el uso de medios electrónicos en Colombia ha tenido un aumento importante los últimos años en los diferentes productos ofrecidos por las entidades financieras, las cuales han diversificado la forma en la que operan, con el fin de facilitar al usuario la recepción o emisión de transferencias, así, los pagos virtuales se convierten en una alternativa con potencial de crecimiento para ampliar la cobertura, además de resultar atractivo para otras entidades gubernamentales en cuanto a recaudos y control de transacciones, basta con contar con una conexión a internet.

En relación con la generación millennials, Cancino et al., (2019) los describen como una de las generaciones influyentes y determinantes del mercado en Colombia debido a que este reacciona ante sus demandas, las cuales se fundamentan en la evolución tecnológica, al haberse adaptado a la era digital. Por su parte, los centennials son considerados por Arango et al., (2019) como la generación que nació en un entorno virtual y tecnológico, por lo cual crece y convive con la tecnología. De allí que puedan estar interesados en usar el internet y los canales móviles web para realzar las compras. En este sentido, Cancino et al. (2019) argumentan que estas generaciones tienen características diferentes de consumo de productos y servicios, por lo que la literatura y las empresas intentan analizarlas para comprender sus necesidades, pero sobre todo cómo suplirlas, en especial las compañías que 
pretenden adoptar la tecnología como estrategia de sostenibilidad en la era digital. Por tanto, la presente investigación pretende analizar los factores más relevantes en la utilización de los pagos móviles en los millennials y centennials. La estructura del texto es la siguiente: i) caracterización de millennials y centennials; ii) descripción del sistema de pagos móviles; iii) modelo propuesto; iv) presentación de la metodología empleada; v) resultados; vi) discusión; vii) conclusiones.

\section{MILLENNIALS Y CENTENNIALS}

La importancia de este estudio en poblaciones como los millennials y centennials radica en que estas generaciones demandan diferentes responsabilidades laborales con altas variabilidades de ingresos de dinero y diferentes motivaciones financieras, se les cataloga como generaciones hiperconectadas al contar con acceso al mundo digital, por tal razón, es la población que más uso puede darle a los servicios financieros a través de los medios virtuales. Como lo muestran Au-Yong-Oliveira, Gonçalves, Martins y Branco (2018), los millennials o generación Y corresponden a una fuerza laboral demográfica cambiante y creciente que incluye a las personas nacidas entre 1981 y 1999 (inicio de la digitalización), y conforman el nuevo mundo móvil y conectado en en el que han redefinido cómo se socializa y se comunica con las demás personas. Por su parte, según Jasso-Peña, Gudiño-Paredes y Tamez-Solís (2019), los centennials son adolescentes que actualmente se encuentran entre los 12 y 18 años (nativos digitales) y se caracterizan por la alta utilización y aprovechamiento de la tecnología, la innovación y la definición de un propio esquema de vida profesional con alta influencia social.

Estas generaciones no están exentas de los trámites financieros habituales del día a día, ya que todas las generaciones requieren realizar pagos, lo que evidencia la relevancia de estudios sobre los diferentes medios disponibles. Juncadella (2004) plantea que los nuevos medios de pagos móviles y virtuales son eficientes y se han trasformado en una línea de negocio fundamental en la captación y retención de clientes en las organizaciones. Para Ruiz-Olivares et al. (2010), los jóvenes se perciben como una población susceptible al riesgo financiero, en especial con la incursión de las Tecnologías de la Información y la Comunicación (TIC), dado que el uso excesivo de estos medios, como el internet y el teléfono móvil, puede ocasionar conductas de dependencia.

\section{SISTEMAS DE PAGOS MÓVILES}

Como lo menciona Tavera y Arias (2012), el internet es una de las tecnologías de información y comunicación más relevante, permite la conexión con múltiples lugares y disminuye la brecha de acceso a información entre las personas. El cierre 
de dicha brecha digital ha ayudado al desarrollo de los países y las regiones a través de los sistemas de pagos móviles y la mayor cobertura que otorga. Esto, según Vahidalizadehdizaj y Leider (2018), debido a la popularidad de la plataforma móvil, que se ha convertido en candidata adecuada para el pago electrónico.

Para Sánchez y Juares (2017) los sistemas de pagos móviles están representados por las operaciones de negocios en línea a través de las plataformas virtuales para realizar todo tipo de transacciones de una forma eficiente. Igualmente, Sathiyavany y Shivany (2018) hacen referencia a la definición del comercio electrónico como el comercio de bienes y servicios mediante redes informáticas. Según Yamakawa, Guerrero y Rees (2013), la banca móvil (mobile banking) es el medio por el cual los aplicativos móviles caracterizados por el ingreso a través de celulares o dispositivos con conexión a internet, ofrecen servicios financieros para las necesidades de los usuarios.

Galán y Venegas (2016) estiman que la facilidad de sistemas electrónicos genera un alto impacto en el mercado financiero al permitir destinar pagos y montos elevados a otros usuarios. Igualmente, las entidades bancarias son las encargadas de realizar un constante monitoreo para garantizar el servicio en las operaciones. De allí que San Martín y Prodanova (2014) argumenten la existencia de factores determinantes en el uso de los dispositivos para efectuar los pagos, como por ejemplo, confianza, satisfacción, propensión a las nuevas tecnologías e influencia social, lo que evidencia que dichos factores influyen en el comportamiento del consumidor para su utilización.

\subsection{Pagos móviles en el contexto colombiano}

Según Gómez-González, Jaramillo-Echeverri y Meisel-Roca (2016), el campo de los medios de pagos móviles en Colombia ha incrementado de manera importante, sobre todo en usuarios con ingresos bajos, pero que tienen las mismas necesidades de realizar operaciones sencillas y necesarias que quienes devengan ingresos muy superiores. Au-Yong-Oliveira et al., (2018) y Jasso-Peña et al., (2019) indican que los millennials y centennials tienen la misma necesidad de utilizar este medio para minimizar el tiempo destinado a estas operaciones. Así, en esta población la penetración de dicho servicio avanza de una forma más rápida por la cercanía con la tecnología, lo que los convierte en consumidores potenciales.

Para Imbachí (2017), el uso de los medios móviles es un fenómeno muy importante y da cuenta del progreso hacia una inclusión financiera, sin embargo, en Colombia los pagos mediante dispositivos móviles no han tenido el crecimiento esperado, debido a las dificultades en plataformas, el alto costo de teléfonos de última generación, la seguridad y la poca capacitación del usuario sobre el uso 
de estos servicios. Benjumea (2016), plantea que los teléfonos móviles generan cada vez más demanda por sus servicios, pero persisten los retos que exige el mercado, en el cual el operador de un medio de pago móvil debe satisfacer las necesidades de los usuarios que las usan, lo cual proporcionaría tranquilidad en términos de accesibilidad, eficiencia y seguridad.

De acuerdo con la Superintendencia Financiera de Colombia (2018), el 79 \% de las operaciones en el 2018 se efectuaron de manera electrónica, un porcentaje considerable. Sumado a ello, se puede evidenciar que la telefonía móvil obtuvo un aumento en el 2018 de 14 \% con respecto al año anterior en cuanto a la utilización de los canales que soportan los sistemas de pagos electrónicos, tal como se evidencia en la tabla 1.

Tabla 1. Número total de operaciones (monetarias y no monetarias)

\begin{tabular}{lcccc}
\hline \multicolumn{1}{c}{ Canales } & 2015 & 2016 & 2017 & 2018 \\
\hline Internet & 1.905 .341 .076 & 2.295 .131 .790 & 2.574 .088 .241 & 3.151 .719 .657 \\
\hline Telefonía móvil & 132.811 .894 & 197.331 .398 & 330.765 .450 & 532.575 .250 \\
\hline Corresponsales bancarios & 147.524 .587 & 184.076 .395 & 234.218 .127 & 325.162 .095 \\
\hline Total & 2.185 .677 .557 & 2.676 .539 .583 & 3.139 .071 .818 & 4.009 .457 .002 \\
\hline
\end{tabular}

Fuente: adaptado de Superintendencia Financiera de Colombia (2018)

\section{FACTORES Y ESTRUCTURACIÓN DEL MODELO}

Algunos antecedentes académicos han estudiado el fenómeno del uso de los medios de pago virtuales (en línea) y de las tecnologías móviles con el objetivo de promover adecuadamente su utilización. Por ejemplo, Ahamad y Pathan (2019) identificaron la seguridad y privacidad de la información como una debilidad en el uso de medios virtuales para efectuar pagos, por lo que plantearon y probaron un protocolo de seguridad como una solución técnica para las transacciones de pago móviles. Luque, Morales y Machado (2017) analizaron aspectos influyentes en la aceptación del dinero electrónico y de las transacciones realizadas por medios de pago en línea (online), destacaron que la desconfianza, actitud negativa, gestión, promoción y seguridad son los factores fundamentales que deben mejorar los proveedores de este servicio. Por otro lado, el estudio de Lu et al., (2017) propuso un modelo para identificar factores determinantes de la adopción de las tecnologías móviles en una región de China y encontraron que: facilidad percibida, utilidad percibida y experiencias prácticas explican la intención de aceptación de estas tecnologías. Lo anterior permitió definir los factores o componentes para esta investigación, los cuales se describen a continuación: 
- Componente 1: actitud hacia el uso (AU). Este componente está asociado a las variables relacionadas con la actitud. Según Chinakidzwa, Mbengo y Nyatsambo (2015), se refiere a los sentimientos, las convicciones o el gusto de una persona por un objeto, idea o individuo. La actitud se validó mediante aspectos como: la percepción de la imagen que las entidades financieras o bancarias generan en el cliente; el interés que manifiesta el cliente a la hora de elegir usar un sistema formal bancario en lugar de recurrir a familiares o amigos; y se preguntó a los usuarios de qué manera influye la imagen de la compañía bancaria cuando cumple con la promesa de proteger los intereses de los clientes. Sobre la actitud de uso, publicaciones como la de Van Deventer, de Klerk, y Bevan-Dye (2018) afirman que es un antecedente del comportamiento real de un individuo.

- Componente 2: conciencia percibida (CO). Las variables agrupadas bajo este factor corresponden a aquellas que evalúan el dominio que los clientes demuestran frente a los temas financieros y de desempeño monetario, además de indagar acerca de la seguridad que les genera a los jóvenes conocer los entes reguladores de las entidades bancarias. Por su parte, Luque, Morales y Machado (2017) indican que la población, en general, tiene a su disposición una serie de servicios financieros en línea que, por su desconocimiento, o no usa o genera una percepción negativa hacia los medios electrónicos.

- Componente 3: confianza percibida (CP). Las variables contenidas en este componente hacen referencia a la percepción de confianza en los dispositivos móviles para realizar transacciones personales en una entidad bancaria y trata de indagar cómo los pagos mediante dispositivos móviles presentan nuevas oportunidades y nuevos riesgos en la utilización para las comunidades de jóvenes. Dicha confianza, según Ahamad, y Pathan (2019), la deben generar las entidades que ofrecen estos servicios, ya que es un aspecto preocupante para los usuarios, dado que en muchas ocasiones tienen miedo de pagar primero antes de recibir el producto.

- Componente 4: credibilidad percibida (CR). En este factor se encuentran las variables encargadas de evaluar todo lo relacionado con la seguridad en la utilización de los teléfonos móviles para realizar operaciones y cómo la trayectoria y buena imagen de una entidad financiera puede influir sobre la decisión de los jóvenes a la hora de adoptar la banca móvil. Para Martínez y Olmedo (2009), un tema de gran importancia es la imagen de las entidades financieras, en este sentido, la reputación de una entidad puede lograr que los usuarios utilicen frecuentemente los productos financieros ofrecidos, en específico, a través de canales virtuales o en línea. 
- Componente 5: experiencia de uso (EU). Asociado a las variables relacionadas con la experiencia ajena o propia que los clientes de la banca móvil consideran a la hora de utilizar los servicios que esta ofrece. En este sentido, Mun, Khalid y Nadarajah (2017) y Lu et al., (2017) señalan que la experiencia propia tiene un gran impacto frente a la decisión de uso de los servicios de pagos móviles.

- Componente 6: intención de uso (SA). Este componente está asociado a las variables relacionadas con la intención de uso. De acuerdo con Khalilzadeh, Ozturk, y Bilgihan (2017), la actitud de los usuarios y la credibilidad o reputación percibida sobre una entidad bancaria o financiera resulta influyente en la intención de utilizar los productos financieros móviles. En adición, Mejía y Quintero (2016) sostienen que tener experiencia de uso en internet aumenta la probabilidad de uso (intención) de los individuos de estos servicios móviles y, en tal caso, si estos usan por primera vez dichos medios de pago, incrementa la probabilidad de que sigan utilizando estos servicios.

\section{METODOLOGÍA}

Esta investigación se basa en un enfoque cuantitativo con un alcance exploratoriodescriptivo orientado a analizar los factores más relevantes en la utilización de los pagos móviles en los millennials y centennials. Se aplica un cuestionario autoadministrado' a 126 millennials y centennials entre los 18 y 35 años en la ciudad de Medellín, como una muestra representativa de la población de estudio. La selección de la muestra se hizo a conveniencia, no probabilístico, con el fin de encuestar a las personas a quienes se tenía más acceso y proximidad. El estudio tuvo como primer criterio de inclusión los jóvenes entre los 18 y 35 años. El 61,11 \% eran mujeres. En cuanto a la distribución por tipo de generación a la que pertencía la población de estudio, el 34,12 \% correspondían a centennials y el 65,87 restante a millennials. El $29,37 \%$ trabajan en empresas dedicadas al comercio, el 26,98 \% en empresas de servicio y el 15,87 \% laboran en el sector financiero. El 27,78\% que resta se distribuye en empresas estatales y otras dedicadas al sector productivo. El cuestionario se compuso de ocho preguntas de selección múltiple y 16 afirmaciones o preguntas en escala de Likert mediante cinco opciones de respuesta (de 1 hasta 5): muy de acuerdo (5), de acuerdo (4), ni en desacuerdo ni de acuerdo (3), en desacuerdo (2) y muy en desacuerdo (1) (ver anexo).

De acuerdo con Pérez y Medrano (2010), el procesamiento de los datos recolectados se realiza con el software estadístico SPSS mediante AFE para explicar

Se trata de un formulario que contiene las preguntas de una encuesta y en el que el propio encuestado, sin intervención de un entrevistador, registra las respuestas. 
los resultados obtenidos en los constructos diseñados en el instrumento. Ello se conoce como la definición o estructuración del modelo teórico, el cual después se valida y evalúa con respecto a su nivel de confiabilidad mediante el análisis factorial confirmatorio (AFC). Para el AFC Anderson, Gerbing, y Hunter (1987) recomiendan analizar primero la validez de cada constructo o factores propuestos, luego para el análisis de confiabilidad, se comparan los constructos a través de los valores de las pruebas corridas para saber si son aceptables, es decir, mayores a 0,6, lo cual garantizaría el criterio de confiabilidad.

De lo anterior, la validez convergente de las escalas de medida utilizadas junto con cada uno de los constructos y el instrumento en general se comprueban a través del método estadístico AFC. Dicho análisis, según Pérez y Medrano (2010), constituye variados procedimientos técnicos para examinar la relación de interdependencia entre variables propuestas con el objetivo de agruparlas de acuerdo con la variabilidad compartida e identificar los factores, dimensiones o conceptos latentes con el propósito de resumir y reducir los datos. Es decir que el AFC es una técnica importante para evaluar la multidimensionalidad de un factor, ya que permite explorar empíricamente y seleccionar aquellas dimensiones que se correlacionan con el conjunto de ítems propuestos para medir el constructo. Por otra parte, Martínez y Martínez (2009) catalogan la validez discriminante como uno de los criterios habituales para evaluar las escalas de medida de constructos latentes en ciencias sociales. En esta fase se afirma que para que unas medidas sean válidas, las escalas de un mismo constructo deben tener una correlación alta entre ellas, y esa correlación debe ser mayor a la que existe con respecto a las medidas propuestas para otro constructo distinto. La confiabilidad (o consistencia) permite verificar la precisión con que el instrumento diseñado mide los factores propuestos sin la presencia de error. En consecuencia, si existe validez convergente y validez discriminante dentro del instrumento, junto con una confiabilidad aceptable, se puede afirmar que el instrumento diseñado presenta la capacidad de evaluar variables fundamentales que tienen influencia sobre las experiencias de la población encuestada.

\section{RESULTADOS}

La primera parte del análisis AFE permitió comprobar que los datos recolectados en el trabajo de campo no presentan información redundante. De acuerdo con Bagozzi y Yi (1988), las cargas factoriales de las variables fueron en su mayoría superiores a 0,6, lo que evidencia la convergencia del modelo. De acuerdo con Pérez y Medrano (2010), el análisis factorial exploratorio (AFE) es un conjunto de métodos estadísticos multivariados de interdependencia cuyo propósito principal es identificar una estructura de factores subyacentes a un conjunto amplio de datos. En este estudio se 
realiza un AFE con el propósito de obtener una estructura base del modelo teórico a presentar y a continuación, validar con el Análisis Factorial Confirmatorio (AFC).

El procedimiento realizado supuso examinar cada conjunto de variables con aplicación de AFE a todo el grupo de variables mediante máxima verosimilitud (ML) como procedimiento de extracción de los factores, y a Varimax como procedimiento de rotación. En segundo lugar, se plantea un modelo en el que se busca validar cinco hipótesis, de acuerdo con los factores obtenidos después de efectuar el AFE; en último lugar se espera corroborar la estructura factorial de cargas estandarizadas obtenidas para cada variable a través de la utilización del AFC. De las variables analizadas, inicialmente se definen seis componentes o factores, en el gráfico 1 se presenta el modelo de hipótesis a validar:

Gráfico 1. Modelo propuesto

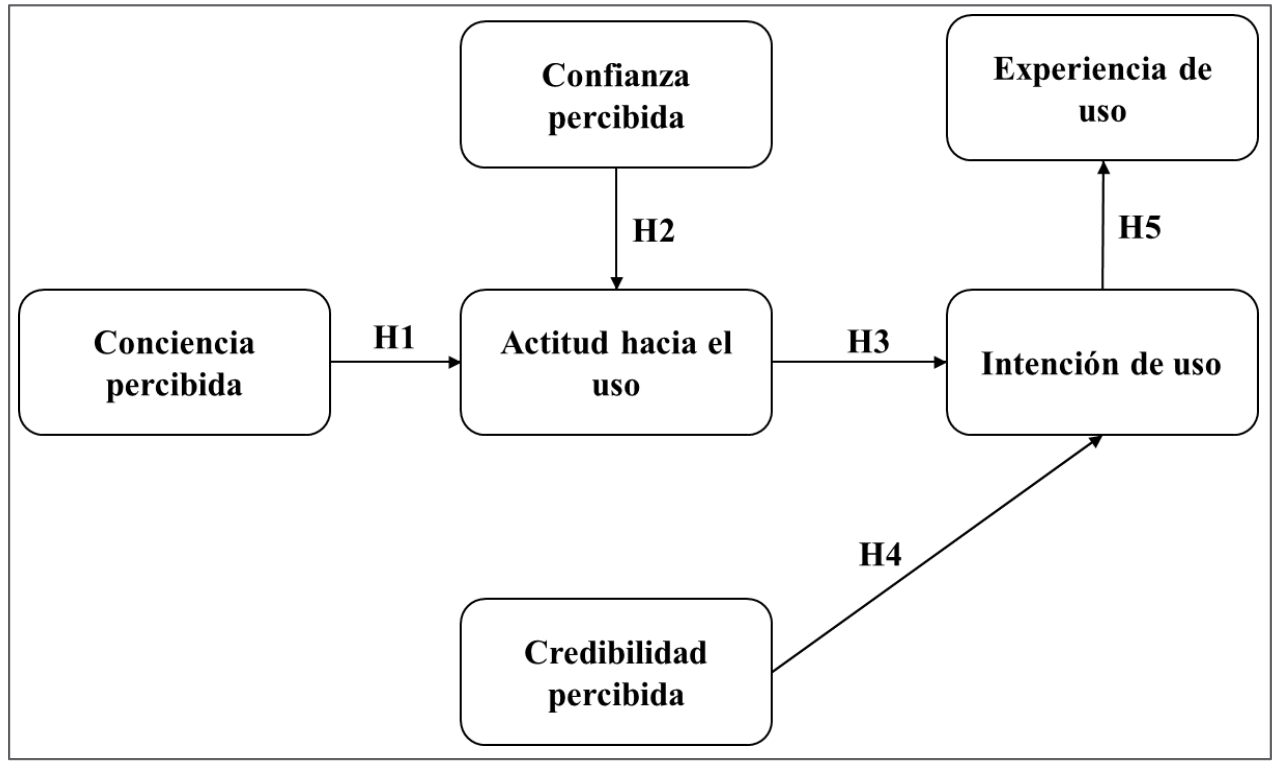

Fuente: elaboración propia a partir de la literatura consultada

Una vez efectuado el AFE se realizó también el AFC, dado que -en palabras de Pérez, Chacón y Moreno (2000) - es una práctica habitual para obtener evidencia de validez de modelos teóricos basados en los resultados del AFE, con esto se demuestra la validez de la estructura factorial previamente obtenida y por consiguiente la validez de las deducciones teóricas inferidas a partir de este, entonces, se procedió a la validación de las escalas en las que se midió la información y la fiabilidad del instrumento de medida utilizado. 


\subsection{Validez convergente}

Como se mencionó con anterioridad, la convergencia prueba el grado en el que la medida de constructos que recogen un mismo concepto está correlacionada, tal como lo afirman Berrios-Riquelme et al., (2019).

La fiabilidad del modelo se valora de dos formas, por un lado, Calvo, Martínez y Juanatey (2013) indican la fiabilidad de los ítems observables y por otro lado, la valoración de la fiabilidad de los constructos. Para determinar su impacto sobre el modelo, algunos autores como Bagozzi y Yi (1988) establecieron que una fiabilidad superior a 0,6 se considerada evidencia de que el modelo es fiable, asimismo, la fiabilidad de los constructos se refiere al grado con que una variable observable refleja un factor, considera aceptable un valor superior a 0,7 . Se tiene entonces que la validez convergente evalúa el grado en que la medida de los ítems que recogen un mismo concepto está correlacionada.

En el presente estudio se evidenció que las cargas factoriales estandarizadas cumplen con los criterios de evaluación establecidos por los autores citados, dado que en general, los constructos arrojaron valores superiores a 0,7 tal y como se muestra en la tabla 2.

Tabla 2. Cargas factoriales estandarizadas de los constructos

\begin{tabular}{|c|c|c|c|}
\hline Constructo & Ítem & Carga factorial estandarizada & $\begin{array}{c}\text { Promedio de cargas factoriales } \\
\text { estandarizadas }\end{array}$ \\
\hline \multirow{3}{*}{ Actitud hacia el uso } & AU1 & 0,720 & \multirow{3}{*}{0,654} \\
\hline & AU2 & 0,579 & \\
\hline & AU3 & 0,664 & \\
\hline \multirow{2}{*}{ Conciencia percibida } & $\mathrm{COl}$ & 0,801 & \multirow{2}{*}{0,801} \\
\hline & $\mathrm{CO} 2$ & 0,801 & \\
\hline \multirow{2}{*}{ Confianza percibida } & $\mathrm{CPl}$ & 0,872 & \multirow{2}{*}{0,872} \\
\hline & $\mathrm{CP} 2$ & 0,872 & \\
\hline \multirow{3}{*}{ Credibilidad percibida } & CR1 & 0,834 & \multirow{3}{*}{0,746} \\
\hline & CR2 & 0,754 & \\
\hline & CR3 & 0,650 & \\
\hline \multirow{3}{*}{ Experiencia de uso } & EU1 & 0,785 & \multirow{3}{*}{0,747} \\
\hline & EU2 & 0,807 & \\
\hline & EU3 & 0,649 & \\
\hline \multirow{3}{*}{ Intención de uso } & IU1 & 0,749 & \multirow{3}{*}{0,740} \\
\hline & IU2 & 0,719 & \\
\hline & IU3 & 0,751 & \\
\hline
\end{tabular}

Fuente: elaboración propia con el software estadístico SPSS 
De forma posterior, la tabla 2 presenta la prueba de esfericidad de Barlett y la medida $\mathrm{KMO}$, debido a que son los estadísticos correspondientes al estudio de la adecuación de la muestra al modelo. Como lo exponen de la Fuente y Justicia (2003), el primer estadístico enunciado hace referencia a una prueba que se emplea para contrastar la hipótesis de que la matriz de correlaciones obtenida no es una matriz de identidad. El valor P debe ser inferior a los niveles críticos 0,05 o 0,01, dado que si el nivel crítico es mayor a 0,05, no es posible rechazar la hipótesis nula de esfericidad y por lo tanto -en palabras de Aldás et al., (2011)-, no se podría asegurar que el modelo factorial sea adecuado para explicar los datos. En vista de que el modelo planteado presenta valores de Bartlett iguales a cero, se puede afirmar que existen correlaciones significativas entre las variables.

Por otro lado, el valor de la medida de adecuación de muestreo de Káiser-MeyerOlkin (KMO) compara las magnitudes de los coeficientes de correlación observados con las magnitudes de los coeficientes de correlación parciales, su valor se da entre 0 y 1, los valores bajos descartan la aplicación de este análisis. Chavarry et al., (2018) califican como aceptables medidas de KMO superiores a 0,5 (KMO > 0,5) e inaceptable la prueba de esfericidad de Bartlett menor a $0.05(<0.05)$.

Como se evidencia en la tabla 3, los coeficientes arrojados por el software SPSS para cada uno de los factores cumple con los criterios mencionados, lo cual indica que es factible realizar la técnica de reducción de datos.

Tabla 3. KMO y prueba de esfericidad de Bartlett

\begin{tabular}{llcc}
\hline \multicolumn{1}{c}{ Constructo } & KMO & Prueba de Bartlett & Cumple criterios \\
\hline Actitud hacia el uso & 0,547 & 0,000 & Sí \\
\hline Conciencia percibida & 0,500 & 0,000 & Sí \\
\hline Confianza percibida & 0,500 & 0,000 & Sí \\
\hline Credibilidad percibida & 0,584 & 0,000 & Sí \\
\hline Experiencia de uso & 0,610 & 0,000 & Sí \\
\hline Intención de uso & 0,634 & 0,000 & Sí
\end{tabular}

Fuente: elaboración propia con el software estadístico SPSS

\subsection{Validez discriminante}

En la presente investigación -de acuerdo con Anderson y Gerbing (1988)-, el análisis de validez discriminante se ejecutó mediante la comprobación de que el intervalo de confianza en la estimación de la correlación entre cada par de factores no contuviera el valor 1. La tabla 4 evidencia que todos los casos cumplen con dicho criterio y de 
esta forma se constata que la validez del instrumento utilizado mide en alto grado aquello que pretendía medir.

Tabla 4. Coeficiente de Spearman

\begin{tabular}{|c|c|c|c|c|c|c|}
\hline & $\begin{array}{l}\text { Actitud hacia } \\
\text { el uso }\end{array}$ & $\begin{array}{c}\text { Conciencia } \\
\text { percibida }\end{array}$ & $\begin{array}{c}\text { Confianza } \\
\text { percibida }\end{array}$ & $\begin{array}{c}\text { Credibilidad } \\
\text { percibida }\end{array}$ & $\begin{array}{l}\text { Experiencia } \\
\text { de uso }\end{array}$ & $\begin{array}{c}\text { Intención de } \\
\text { uso }\end{array}$ \\
\hline Actitud hacia el uso & 1 & & & & & \\
\hline Conciencia percibida & $\begin{array}{c}{[-0,167} \\
0,197]\end{array}$ & 1 & & & & \\
\hline Confianza percibida & $\begin{array}{l}{[0,026} \\
0,403]\end{array}$ & $\begin{array}{l}{[0,001 ;} \\
0,370]\end{array}$ & 1 & & & \\
\hline Credibilidad percibida & $\begin{array}{c}{[-0,064 ;} \\
0,309]\end{array}$ & $\begin{array}{l}{[0,014} \\
0,379]\end{array}$ & $\begin{array}{l}{[0,120} \\
0,464]\end{array}$ & 1 & & \\
\hline Experiencia de uso & $\begin{array}{c}{[0,071} \\
0,415]\end{array}$ & $\begin{array}{c}{[-0,087} \\
0,289]\end{array}$ & $\begin{array}{c}{[0,009} \\
0,376]\end{array}$ & $\begin{array}{l}{[0,014 ;} \\
0,363]\end{array}$ & 1 & \\
\hline Intención de uso & $\begin{array}{c}{[-0,030} \\
0,313]\end{array}$ & $\begin{array}{l}{[0,179} \\
0,544]\end{array}$ & $\begin{array}{c}{[0,096} \\
0,462]\end{array}$ & $\begin{array}{l}{[0,157} \\
0,492]\end{array}$ & $\begin{array}{l}{[0,019} \\
0,365]\end{array}$ & 1 \\
\hline
\end{tabular}

Fuente: elaboración propia con apoyo del software estadístico SPSS

Frías (2019) señala que la fiabilidad de la consistencia interna del instrumento se estima por medio del alfa de Cronbach, en vista de que es una herramienta en la que se asume que los ítems (escala tipo Likert) miden un mismo constructo y están altamente correlacionados. En lo que respecta a su interpretación, se tiene que Nunnally y Bernstein (1994) afirman que si el valor del alfa de Cronbach es más cercano a 1, la consistencia interna de los ítems es mayor, puesto que la prueba alcanza valores positivos comprendidos entre 0 y 1 , en la que 0 indica ausencia total de consistencia interna y 1, la redundancia total entre los ítems. Sin embargo, en las primeras fases de la investigación un valor de fiabilidad de 0,6 o 0,5 puede ser suficiente.

En la tabla 5 se observa que el instrumento de medida parece tener una adecuada confiabilidad de la consistencia interna de la escala de medida, pues todos los alfa de Cronbach se encuentran entre el rango de valores recomendados por los autores mencionados.

Los resultados del análisis confirmatorio evidencian un modelo factorial sus tentable para el análisis fundamentado en la identificación de variables que inciden en la adopción de la banca móvil en jóvenes millennials y centennials de Medellín. La presencia de validez convergente y validez discriminante en el instrumento, junto con una confiabilidad aceptable, ratifica que el instrumento evalúa variables 
fundamentales que inciden directa o indirectamente sobre las experiencias de los estudiantes encuestados en la investigación.

Tabla 5. Índice de fiabilidad. Alfa de Cronbach

\begin{tabular}{lc}
\hline \multicolumn{1}{c}{ Constructo } & Alfa de Cronbach \\
\hline Actitud hacia el uso & 0,645 \\
\hline Conciencia percibida & 0,796 \\
\hline Confianza percibida & 0,878 \\
\hline Credibilidad percibida & 0,792 \\
\hline Experiencia de uso & 0,782 \\
\hline Intención de uso & 0,772 \\
\hline
\end{tabular}

Fuente: elaboración propia con el software estadístico SPSS

\subsection{CONTRASTE DE HIPÓTESIS}

Abascal y Grande (2005) exponen que para la validación se recogen las diversas hipótesis planteadas y se mide su grado de asociación por medio del coeficiente de correlación V de Cramer, el cual arroja el grado de asociación entre las variables tenidas en cuenta para la formulación de hipótesis. Los valores se encuentran entre 0 y 1 , y se alcanzan valores extremos cuando existe concordancia o discordancia perfecta; los valores próximos a 0 indican ausencia de asociación. En la tabla 6 se encuentran los valores obtenidos para el estadístico de asociación analizado y el modelo utilizado. Los coeficientes de asociación calculados para las relaciones hipotéticas del modelo no presentan valores significativos, esto es, una baja correlación entre las variables evaluadas en el análisis, no obstante, es posible que los valores de asociación estén subestimados debido al número de ítems observables que componen los constructos del análisis.

\section{Tabla 6. Contraste de hipótesis}

\begin{tabular}{clcl}
\hline Hipótesis & \multicolumn{1}{c}{ Constructo } & V de Cramer & \multicolumn{1}{c}{ Constructo } \\
\hline H1 & Conciencia percibida & 0,152 & Actitud hacia el uso \\
\hline H2 & Confianza percibida & 0,226 & Actitud hacia el uso \\
\hline H3 & Actitud hacia el uso & 0,174 & Intención de uso \\
\hline H4 & Credibilidad percibida & 0,245 & Intención de uso \\
\hline H5 & Intención de uso & 0,451 & Experiencia de uso \\
\hline
\end{tabular}

Fuente: elaboración propia con el software estadístico SPSS 
Los estadísticos se tomaron del software SPSS y ubicados en una tabla de factores cruzados, para que fuera posible observar el grado de asociación entre las variables que hacían parte de las hipótesis y las que no lo hacían y al mismo tiempo, para corroborar que entre los demás constructos no se presentara un nivel de asociación alto. La tabla 7 evidencia todas las relaciones establecidas entre las variables del modelo propuesto.

Tabla 7. Coeficiente V de Cramer, unidireccional

\begin{tabular}{lcccccc}
\hline & $\begin{array}{c}\text { Actitud hacia } \\
\text { el uso }\end{array}$ & $\begin{array}{c}\text { Conciencia } \\
\text { percibida }\end{array}$ & $\begin{array}{c}\text { Confianza } \\
\text { percibida }\end{array}$ & $\begin{array}{c}\text { Credibilidad } \\
\text { percibida }\end{array}$ & $\begin{array}{c}\text { Experiencia } \\
\text { de uso }\end{array}$ & $\begin{array}{c}\text { Intención de } \\
\text { uso }\end{array}$ \\
\hline Actitud hacia el uso & 1 & & & & & \\
\hline Conciencia percibida & 0,152 & 1 & & & & \\
\hline Confianza percibida & 0,226 & 0,215 & 1 & & & \\
\hline Credibilidad percibida & 0,223 & 0,36 & 0,227 & 1 & 1 \\
\hline Experiencia de uso & 0,213 & 0,21 & 0,26 & 0,166 & 0,451 & 1 \\
\hline Intención de uso & 0,174 & 0,34 & 0,259 & 0,245 & & \\
\hline
\end{tabular}

Fuente: elaboración propia con el software estadístico SPSS

El gráfico 2 presenta el modelo obtenido después de efectuar el AFE con sus respectivos valores de asociación entre las variables, las líneas simbolizan las hipótesis nulas que se validaron en la investigación.

Gráfico 2. Modelo propuesto, V de Cramer

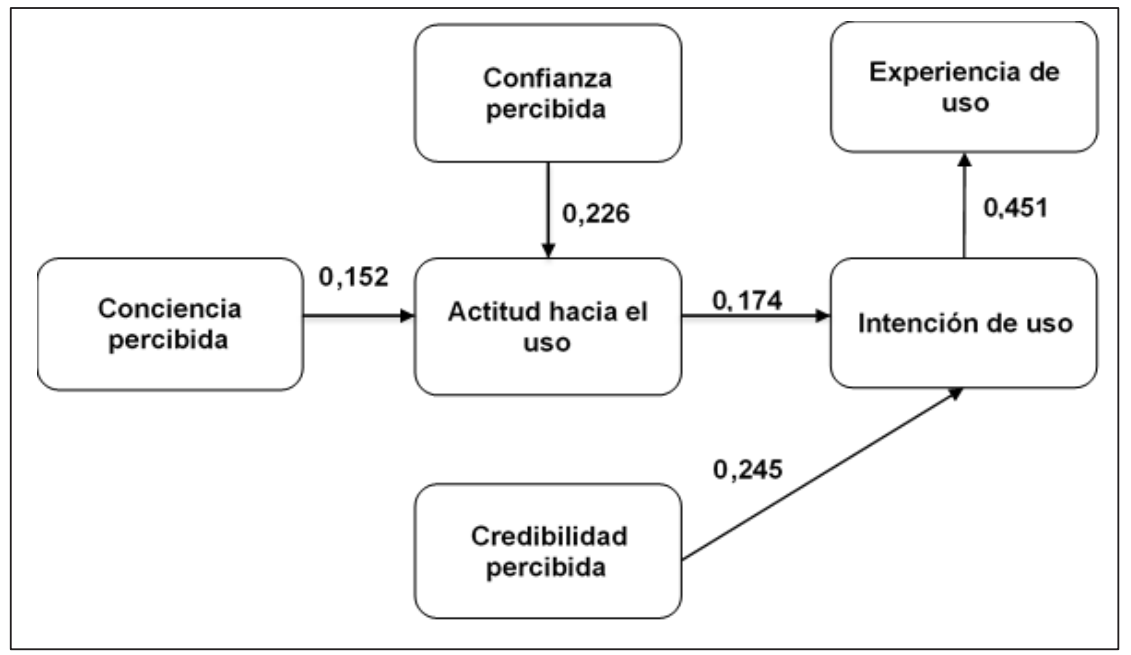

Fuente: elaboración propia 


\section{DISCUSIÓN}

Uno de los aspectos relevantes al evaluar la utilización de los pagos móviles en los milennialls y centenialls está relacionado con la experiencia de uso que ha tenido su círculo cercano, en otras palabras, los jóvenes buscan en experiencias ajenas entender si el servicio que piensan adquirir es útil y práctico, de hecho, encontrar que una determinada tecnología es práctica y eficaz ejerce una influencia significativa en las personas en cuanto a la intención de uso de dicha tecnología, tal como lo mencionan Jaradat y Faqih (2014), quienes exponen que los consumidores potenciales son aquellos que perciben calidad y utilidad del sistema de pago móvil. En el modelo propuesto se evidencia una relación fuerte (valor distante de cero) entre los constructos experiencia de uso e intención de uso $(0,451)$. Esta correlación demuestra que generar una buena experiencia en estas generaciones se convierte en la herramienta clave para cambiar o mejorar la percepción que los jóvenes tienen de la adopción de la banca móvil.

La actitud hacia el uso no representa en esta oportunidad un constructo moderador de la intención de uso $(0,174)$, esto se puede deber a que el tema de adopción de banca móvil tiene una fuerte tendencia a revisar aspectos más puntuales y tangibles como la calidad de la infraestructura de red, el correcto funcionamiento de las instalaciones de cómputo, incluso se le da mayor importancia a los centros de servicio, dado que estos se convierten en un activo estratégico fundamental para las entidades financieras y marcan el camino hacia la calidad en la atención y una diferenciación, elementos fundamentales a la hora de analizar los factores sociales que influyen en la intención de emplear pagos móviles. Ello contrasta con otras investigaciones como las de Van Deventer, de Klerk y Bevan-Dye (2018) en las cuales la actitud tiene influencia significativa y positiva en el comportamiento de uso de la banca móvil.

Para Amin (2007), la credibilidad percibida compuesta de dos elementos importantes - privacidad y seguridad- hace referencia a la protección de la información, dado que se ha encontrado que el miedo a la falta de seguridad es uno de los factores por los cuales los jóvenes desisten del uso de la banca móvil y tiene una relación mediana con la intención de uso $(0,245)$. En conclusión, el constructo de conciencia percibida concuerda con la definición de Fonchamnyo (2013) al referirse al grado en que los usuarios están informados sobre la existencia de la nueva innovación tecnológica; encuentra que es un constructo que no requiere ser moderado por la actitud hacia el uso $(0,152)$ y que se relaciona directamente con la intención de uso $(0,340)$.

Las implicaciones prácticas de este estudio se orientan hacia las entidades que desarrollan la banca móvil, quienes deben diseñar estrategias para aumentar el 
número de clientes que utilizan el sistema de pagos móviles, al considerar responder a las necesidades de los clientes millennials y centennials. Por otro lado, los constructos propuestos se extrajeron de la literatura -algunos de estos basados en la teoría de la psicología social de Boonsiritomachai y Pitchayadejanant (2017)-, por lo que los resultados de esta investigación pueden explicar la probabilidad de utilización en estas dos generaciones (clientes) de los medios de pago móvil. Además, el valor agregado de los resultados está en el aporte teórico práctico que se genera para Colombia sobre la comprensión del comportamiento frente a aspectos financieros y tecnológicos, en especial para la ciudad de Medellín, donde se observa que poblaciones como los millennials poseen características generacionales muy diferentes al resto de la generación $Y$ de Latinoamérica y el mundo, según lo explican Posada y White (2016), las cuales dependen de las condiciones culturales, económicas y políticas del país.

En el contexto colombiano los resultados obtenidos en la presente investigación son relevantes para los millennials y centennials de la ciudad de Medellín, debido a que según el Departamento Administrativo Nacional de Estadística (DANE) (2019), en Antioquia los jóvenes conforman el 27 \% de la población, por lo que es pertinente llevar a cabo estudios sobre sus comportamientos y tendencia al uso de dispositivos electrónicos. Se ha evidenciado también que la ciudad tiene la intención de potencializar la cuarta revolución industrial y el uso de las TIC (Dinero, 2019; Mercado, 2019), por lo que las variables analizadas en este estudio resultan coherentes con investigaciones entre la población juvenil en Medellín de autores como Ospina et al. (2019), en las cuales las conductas de los jóvenes de entornos virtuales dependen de las experiencias, confianza e influencia social.

\section{CONCLUSIONES}

La aceptación de los pagos móviles para efectuar pagos virtuales depende de la aceptación de los clientes y más para las generaciones reconocidas como nativos digitales, los cuales lideran el cambio encaminado hacia las nuevas tecnologías. El enfoque metodológico utilizado en esta investigación verificó y probó empíricamente la capacidad explicativa del modelo planteado para la utilización de los medios de pagos virtuales y concluyó que para las generaciones millennials y centennials, la experiencia en el uso y la conciencia percibida de la banca móvil tienen una influencia directa y positiva en sus actitudes hacia el uso de estos medios de pago.

Los resultados obtenidos son relevantes científicamente porque aportan un estudio empírico para contribuir a llenar el vacío de literatura sobre el comportamiento financiero y las preferencias tecnológicas en las generaciones millennials 
y centennials. En cuanto a las implicaciones prácticas, pueden ser utilizados por los profesionales, comerciantes, estrategas, formuladores de políticas y analistas financieros para comprender mejor el comportamiento de la banca móvil de los clientes de las generaciones vinculadas a la tecnología, con el fin de desarrollar una formulación efectiva de estrategias que promuevan una mayor aceptación del cliente por los medios de pagos virtuales.

Con respecto a los futuros trabajos, se recomienda analizar estas generaciones (millennials y centennials) con base en otro tipo de variables financieras como ingresos, capacidad de endeudamiento y hábito de ahorro para dar cuenta de los efectos sobre el uso de las tecnologías móviles para la administración y gestión de sus finanzas personales, lo cual podría explicar la intención de uso de la banca móvil. Al mismo tiempo, se recomienda en estas generaciones, en el contexto colombiano, identificar si haber recibido formación o contar con educación en el área de sistemas de información y la computación puede influir en la decisión de uso de los medios de pago por los cuales se efectúa el consumo, como lo son las plataformas, aplicativos y tecnologías móviles. De esta manera se podrá hacer una elección apropiada de la mejor estrategia o combinación de ellas que maximice la adopción de estos medios virtuales.

\section{BIBLIOGRAFÍA}

Abascal, Elena y Grande, Ildefonso (2005). Análisis de encuestas. Madrid: ESIC Editorial. 291 p.

Acevedo-Prins, Natalia, y Jiménez-Gómez, Luis Miguel (2015). Índice para la medición de la competitividad en Colombia. En: Revista CEA, Vol. 1, N. 2, p. 109-121.

Aghili, Seyed Farhad y Mala, Hamid (2019). Security analysis of an ultra】lightweight RFID authentication protocol for m-commerce. En: International Journal of Communication Systems,

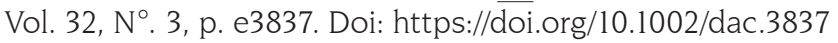

Ahamad, Shaik Shakeel y Pathan, Al-Sakib Khan (2019). Trusted service manager (TSM) based privacy preserving and secure mobile commerce framework with formal verification. En: Complex Adaptive Systems Modeling, Vol 7, N. 1, p. 1-18. Doi:10.1186/s40294-019-006 $\overline{4-Z}$

Alalwan, Ali Abdallah; Dwivedi, Yogesh; Rana, Nripendra y Algharabat, Raed (2018). Examining factors influencing Jordanian customers' intentions and adoption of internet banking: Extending UTAUT2 with risk. En: Journal of Retailing and Consumer Services, Vol. 40, p. 125-138. https://doi.org/10.1016/j.jretconser.2017.08.026

Aldás, Joaquín; Lassala, Carlos; Ruiz, Carla y Sanz, Silvia (2011). Análisis de los factores determinantes de la lealtad hacia los servicios bancarios online. En: Cuadernos de Economía y Dirección de la Empresa, Vol. 14, N.1, p. 26-39. Doi: https://doi.org/10.1016/j.cede.2011.01.003

Amin, Hanudin (2007). Internet Banking Adoption Among Young Intellectuals. En: Journal of Internet Banking and Commerce. Vol. 12, $\mathrm{N}^{\circ}$.3. p. 1-13. 
Anderson, James y Gerbing, David (1988). Structural equation modeling in practice: A review and recommended two-step approach. En: Psychological bulletin, Vol. 103, N.3, p. 411 423.

Anderson, James; Gerbing, David y Hunter, John (1987). On the assessment of unidimensional measurement: Internal and external consistency, and overall consistency criteria. En: Journal of marketing research, Vol. 24, N. 4, p. 432-437.

Arango, Carlos Andrés; Camelo, Carolina; Huertas, María; Sánchez, Catherine; Sánchez, Vladimir; Sojo, Jairo y Arango, Carlos (2019). 1218 Centennials: Generación Sin Etiquetas. Bogotá, Colombia: Fundación Universidad de Bogotá Jorge Tadeo Lozano, 150p.

Arango, Carlos Alberto; Zarate-Solano; Hector Manuel y Suárez-Ariza, Nicolás. (2017). Determinantes del Acceso, Uso y Aceptación de Pagos Electrónicos en Colombia. En: Borradores de Economía. №. 999, p. 1-59.

Au-Yong-Oliveira, Manuel; Gonçalves, Ramiro; Martins, José y Branco, Frederico (2018). The social impact of technology on millennials and consequences for higher education and leadership. En: Telematics and Informatics, Vol. 35, N. 4, p. 954-963. Doi: https://doi.org/10.1016/j. tele.2017.10.007

Bagozzi, Richard y Yi, Youjae (1988). On the evaluation of structural equation models. En: Journal of the academy of marketing science, Vol. 16, N. 1, p. 74-94. Doi: https://doi.org/10.1007/ BF02723327

Banu, Adriana; Ganagavalli y Ramsundar, Bharath (2018). OR Code Based Shopping with Secure Checkout for Smartphones. En: Journal of Computational and Theoretical Nanoscience, Vol. 15, N. 5, p. 1545-1550.

Benjumea, Ana María (2016). Los retos de la banca móvil en la inclusión financiera en Colombia. Tesis para optar al título de magíster en Administración (MBA). Maestría en Administración (MBA), Universidad Eafit, Colombia. 40p.

Berrios-Riquelme, José; Frías-Navarro, Dolores; Pascual-Soler, Marcos y Muzatto-Negron, Paola (2019). Escala de satisfacción con la vida: propiedades psicométricas de inmigrantes en España. En: Revista de ciencias sociales, Vol. 25, №. 1, p. 130-142.

Boonsiritomachai, Waranpong y Pitchayadejanant, Krittipat (2017). Determinants affecting mobile banking adoption by generation Y based on the Unified Theory of Acceptance and Use of Technology Model modified by the Technology Acceptance Model concept. En: Kasetsart Journal of Social Sciences. Vol. 40, N. 2, p. 349-358. Doi: https://doi.org/10.1016/j. kjss.2017.10.005

Calvo-Porral, Cristina; Martínez-Fernández, Valentín-Alejandro y Juanatey-Boga, Oscar (2013). Análisis de dos modelos de ecuaciones estructurales alternativos para medir la intención de compra. En: Revista Investigación Operacional, Vol. 34, Nº. 3, p. 230-243.

Cancino, Mónica; Chaparro, Alberto Daniel; Puentes, Natalia; Rojas, Lady Viviana y Pérez, Rafael (2019). Factores Determinantes Para La Decisión de Compra Por Plataformas Digitales Para Los Millennials Ubicados En Bogotá D. C. Bogotá, Colombia: Universidad EAN, 62p.

Chavarry, Edward Anthony; Sosa, Irvin David; Díaz-Vélez, Cristian y Salazar-Zuloeta, Jaime Israel (2018). Validación de Un Instrumento Para Medir Nivel de Conocimiento de Médicos Del 
Primer Nivel de Atención de Salud Sobre Los Signos de Alarma Del Dengue. En: Rev Hisp Cienc Salud. Vol. 3, N. 4, p. 121-28.

Chinakidzwa, More; Mbengo, Pinigas y Nyatsambo, Marcelene (2015). Mobile Money in Rural Areas of Zimbabwecase of Mudzi District. En: International Journal of Scientific y Engineering Research, Vol 6, N. 2, p. 128-132.

Davis, Fred (1989). Perceived usefulness, perceived ease of use, and user acceptance of information technologies. En: MIS Quarterly, Vol. 13, N. 3, p. 319-340. Doi: https://doi. org/10.2307/249008

De la Fuente, Jesus y Justicia, Fernando (2003). Escala de estrategias de aprendizaje ACRAAbreviada para alumnos universitarios. En: Electronic journal of research in educational psychology, Vol. 1, №. 2, p. 139-158.

Departamento Administrativo Nacional de Estadística (DANE) (2019). Índices Demográficos. Bogotá, Colombia: DANE.

Dinero (2019).Inauguran en Medellín el primer Centro para la Cuarta Revolución Industrial de Hispanoamérica. Bogotá, Colombia: Dinero.

Fonchamnyo, Dobdinga Cletus (2013). Customers' perception of E-banking adoption in Cameroon: An empirical assessment of an extended TAM. En: International Journal of Economics and Finance, Vol. 5, No.1, p. 166 176. Doi: http://dx.doi.org/10.5539/ijef.v5n1pl66

Frías-Navarro, Dolores (2019). Apuntes de consistencia interna de las puntuaciones de un instrumento de medida. Valencia, España: Universidad de Valencia, 13p.

Galán, Javier y Venegas, Francisco. (2016). Impacto de los medios electrónicos de pago sobre la demanda de dinero. En: Investigación Económica, Vol. 75, N.295, p. 93-124. Doi: https:// doi.org/10.1016/j.inveco.2016.03.003

Gómez-González, José Eduardo; Jaramillo-Echeverri, Juliana y Meisel-Roca, Adolfo (2016) El Uso de Efectivo y Tarjetas Débito y Crédito En Colombia. En Borradores de Economía; $N^{\circ} .950$, p. $1-29$.

Imbachí, Jair Fernando (2017). M-commerce. El comercio electrónico móvil y los pagos a través de dispositivos móviles. En: Con-texto, №. 46, p. 117-141. Doi: https://doi.org/10.18601/01236458. n46.08

Jaradat, Mohammed-Issa y Faqih, Khaled (2014). Investigating the Moderating Effects of Gender and Self-Efficacy in the Context of Mobile Payment Adoption: A Developing Country Perspective. En: International Journal of Business and Management, Vol. 9, No.11, p.147-169. Doi: http://dx.doi.org/10.5539/ijbm.v9n11p147

Jasso-Peña, Felipe de Jesus; Gudiño-Paredes, Sandra y Tamez-Solís, José Porfirio (2019). Centennials, ciudadanos globales y digitales Centennials. En: Praxis, Vol. 15, № 1, p. 11-23.

Juncadella, Javier Santomá (2004). Nuevos medios de pago electrónicos: hacia la desintermediación bancaria. En: Revista de Economía, №. 813, p. 101-114.

Khalilzadeh, Jalayer; Ozturk, Ahmet Bulent y Bilgihan, Anil (2017). Security-related factors in extended UTAUT model for NFC based mobile payment in the restaurant industry. En: Computers in Human Behavior, Vol. 70, p. 460 474.Doi: https://doi.org/10.1016/j.chb.2017.01.001 
Li, Songnong; Hu, Xiaorui; Zhang, Yu; Dong, Wei; Ye, Jun y Sun, Hongliang (2018). Research on Offline Transaction Model in Mobile Payment System. En: Lecture Notes in Electrical Engineering, Vol. 542 p. 1815-1820. Doi: https://doi.org/10.1007/978-981-13-3648-5_235

Lu, June; Wei, June; Yu, Chun-Sheng y Liu, Chang (2017). How do post-usage factors and espoused cultural values impact mobile payment continuation? En: Behaviour y Information Technology, Vol. 36, N. 2, p. 140-164.

Luque, Arturo; Morales, Tania y Machado, Libertad (2017). Dinero Electrónico, Desconocimiento Y Resistencias: el caso de Ambato y Machala en Ecuador. En: Revista Ciencias Pedagógicas E Innovación, Vol. 5, Nº. 3. p.128-139. Doi: https://doi.org/10.26423/rcpi.v5i3.210

Martínez, Inocencia María y Olmedo, Isabel. (2009). La medición de la reputación empresarial: problemática y propuesta. En: Investigaciones Europeas de Dirección y Economía de la Empresa, Vol. 15, N.2, p. 127-142. Doi: https://doi.org/10.1016/S1135-2523(12)60093-8

Martínez-García, José Antonio y Martínez-caro, Laura (2009). La validez discriminante como criterio de evaluación de escalas: ¿teoría o estadística. En: Universitas Psychologica, Vol. 8, N.1, p. 27-36.

Medina, Deysi. (2011). Bancarización en Milagro: Utilización de servicios electrónicos. En: Revista Ciencia Unemi, N. 6, p. 16-27.

Medrano, María Luisa y Díez, Luis. (2010). El comercio y las finanzas de la juventud medios. En: Revista de Estudios de juventud, $N^{\circ}$. 88. p 165-181.

Mejía, Manuel Antonio y Quintero, Wilmar. (2016). Determinantes del uso de la banca electrónica en Colombia. En: Revista Finnova: Investigación e Innovación Financiera y Organizacional, Vol. 2, No.3, p. $\overline{15}-26$.

Mercado, Alejandro (2019).Los 4 retos que trae la Cuarta Revolución Industrial a Medellín. Medellín, Colombia: El Tiempo.

Moreno-Agudelo, Jorge. Andrés, Y Valencia-Arias, Alejandro (2017). Factores implicados en la adopción de software libre en las Pyme de Medellín. En: Revista CEA, Vol. 3 N‥ 6, p. 55-75. Doi: https://doi.org/10.22430/24223182.673

Mun, Yeow Pooi; Khalid, Haliyana y Nadarajah, Devika (2017). Millennials' perception on mobile payment services in Malaysia. En: Procedia Computer Science, Vol. 124, p. 397-404. Doi: https://doi.org/10.1016/j.procs. 2017.12 .170

Nie, Jin y Amarayoun, Wipapach. (2018). The Factors Influence the Intention Use of Mobile Payment in Thailand E-Commerce. En: Information Science and Control Engineering. p. 561-568. Doi: 10.1109/ICISCE.2018.00122

Nunnally, Jum y Bernstein, Ira. (1994). Psychometric Theory (3ra ed.). New York: McGraw-Hill, 752p.

Ospina, Carolina; Araque, Isabel; Ordoñez, Samir y Torres, Isabel Cristina (2019). Caracterización de los adolescentes en la ciudad de Medellín. En: Revista Publicuidad, Vol. 8, Nº 1, p. 77-90. Doi: https://doi.org/http://dx.doi.org/10.18566/publicuidad.v08n01.a05

Pérez, José Antonio; Chacón, Salvador y Moreno, Rafael (2000). Validez de constructo: el uso del análisis factorial exploratorio-confirmatorio para obtener evidencias de validez. En Psicothema, Vol. 12, N.2, p. 442-446. 
Pérez, Edgardo y Medrano, Leonardo. (2010). Análisis factorial exploratorio: bases conceptuales y metodológicas. En: Revista Argentina de Ciencias del Comportamiento, Vol. 2, Nº.1, p. 58-66.

Posada, María Camila y White, Mariana (2016). Tipificación del consumo de la generación "Millennial" en Medellín en los estratos 4, 5 y 6. Tesis para optar el título de Ingeniera Administradora, Ingeniería administrativa Universidad EIA, Colombia. 158p.

Ruiz-Olivares, Rosario; Lucena, Valentina; Pino, M. José y Herruzo, Javier (2010). Análisis de comportamientos relacionados con el uso/abuso de internet, teléfono móvil, compras y juego en estudiantes universitarios. En: Adicciones, Vol. 22, N. 4, p. 301-310. Doi: http:// dx.doi.org/10.20882/adicciones.171

San Martín, Sonia y Prodanova, Jana (2014). ¿Qué factores fomentan la compra por impulso en el comercio móvil? En: Revista Española de Investigación de Marketing ESIC, Vol. 18 N. 1, p. 32-42. Doi: https://doi.org/10.1016/S1138-1442(14)60004-8

Sánchez, Javier y Juares, Fernando (2017). La adopción del comercio electrónico en las pymes: una revisión de la literatura exploratoria. En: Novum revista de ciencias sociales aplicadas, $\mathrm{N}^{\circ} .7$, pp. 78-94.

Sathiyavany, Nai y Shivany, Shanmugathas (2018). E-Banking Service Qualities, E-Customer Satisfaction, and e-Loyalty: A conceptual Model. En: The International Journal of Social Sciences and Humanities Invention, Vol. 5, N. 6, p. 4808 4819. Doi: https://doi.org/10.18535/ ijsshi/v5i6.08

Shaw, Norman y Sergueeva, Kasenia (2019). The non-monetary benefits of mobile commerce: Extending UTAUT2 with perceived value. En: International Journal of Information Management, Vol. 45, p. 44-55. Doi: https://doi.org/10.1016/j.ijinfomgt.2018.10.024

Superintendencia Financiera de Colombia (2018). Informe de operaciones. Bogotá, Colombia: Superintendencia Financiera de Colombia, 38p.

Tavera, Juan Fernando y Arias, José Enrique (2012). Internet Móvil: Aceptación tecnológica para el cierre de la brecha digital en Colombia. En: Perfil de Coyuntura Económica, №. 19, p. 139-156.

Thammarat, Chalee y Kurutach, Werasak (2019). A lightweight and secure NFC-base mobile payment protocol ensuring fair exchange based on a hybrid encryption algorithm with formal verification. En: International Journal of Communication Systems, Vol. 32, №. 12, p. e3991.

Trojanowski, Mariusz y Kułak, Jacek (2018). Understanding mobile purchase intentions in poland: extension of the technology acceptance model. En: Information Systems Architecture and Technology, Vol. 852 p. 334-344. Doi: https://doi.org/10.1007/978-3-319-99981-4_ 31

Vahidalizadehdizaj, Mohammad y Leider, Avery (2018). Mobile Payment Protocol 3D (MPP 3D) by Using Cloud Messaging. En: In Information Technology-New Generations, Vol. 558, p. 23-31. Doi: https://doi.org/10.1007/978-3-319-54978-1_ 4

van Deventer, Marco., de Klerk, Natasha, y Bevan-Dye, Ayesha (2018). Influence of perceived ease of use and perceived relative advantage on generation y students'attitudes towards and usage behaviour of mobile banking in South Africa. En: International Journal of eBusiness and eGovernment Studies, Vol. 10, N. 1, p. 18-33. 
Yamakawa, Peter; Guerrero, Carlos y Rees, Gareth (2013). Factores que influyen en la utilización de los servicios de banca móvil en el Perú. En: Universidad E Empresa, №. 25, p. 131-149.

Yang, Jen-Ho y Lin, Pei-Yu (2018). A New Third-party Payment Scheme with Anonymity for Mobile Commerce. En: Journal of Internet Technology, Vol. 19, N. 6, p. 1929-1934.

Yeh, Kuo-Hui; Su, Chunhua; Hou, Jia-Li; Chiu, Wayne y Chen, Chien-Ming (2018). A Robust Mobile Payment Scheme With Smart Contract-Based Transaction Repository. En: IEEE Access, Vol. 6, p. 59394-59404. Doi: 10.1109/ACCESS.2018.2874021 


\section{ANEXO \\ CUESTIONARIO DEL ESTUDIO CUANTITATIVO}

\section{El objetivo de esta encuesta es analizar factores asociados a la utilización de los medios de pago móviles en millennials y centennials. Las respuestas obtenidas serán confidenciales y serán objeto de estudio; por tanto, solo será para fines académicos.}

En las preguntas de la 1 a la 8, seleccione con una "X" sus respuestas.

1. Por favor indique su sexo.

$\square$ Masculino $\square$ Femenino

2. Rango de edad:
a. 18 a 20 años
b. 20 a 25 años.
c. 25 a 30 años
d. 30 a 35 años

3. Grado de escolaridad
a. Primaria
b. Secundaria
c. Técnico
d. Tecnología
e. Profesional
f. Posgrado

4. ¿Cuál es el sector en que labora?
a. Industrial
b. Entidades públicas
c. Comercial
d. Independiente e. Financiero
f. Servicios

5. ¿Cuáles aplicaciones conoce? Seleccione con una "X" la(s) respuesta(s)
a. Aplicaciones bancarias (Apps)
b. PSE
c. Paypal
d. Google Wallet
e. Otra. ¿Cuál?

6. ¿Cuáles de las siguientes opciones le genera más seguridad para realizar sus pagos? Seleccione con una "X" la(s) respuesta(s)
a. Trámite presencial ante entidad bancaria
b. Dispositivo móvil
d. Ingreso directo desde computador
e. Otra. ¿Cuál?

7. ¿De estos servicios, ¿cuáles son los más utilizados al acceder desde el dispositivo móvil? Seleccione con una "X" la(s) respuesta(s)

a. Transacciones b. Consultas de saldo c. Pagos de facturas d. Compras

e. Otra. ¿Cuál?

8. ¿Por qué razón realiza sus pagos u otra operación mediante el teléfono móvil? Seleccione con una "X" la(s) respuesta(s)

a. Alta cobertura en zonas sin instuticón financiera b. No neceista dinero en efectivo
c. Ahorro en tiempo
d. Ahorro en costo por desplazamiento
e. Por seguridad

f. Costo bajo asociado a operación g. Otra. ¿Cuál?

9. En las siguientes afirmaciones, seleccione con una "X" el nivel de acuerdo o desacuerdo que tenga con cada una de ellas, siendo 1) totalmente en desacuerdo; 2) en desacuerdo; 3) ni de acuerdo, ni en desacuerdo; 4) de acuerdo: 5) totalmente de acuerdo.

\begin{tabular}{|c|l|l|l|l|l|l|}
\hline \multicolumn{2}{|c|}{ Afirmaciones } & 5 & 4 & 3 & 2 & 1 \\
\hline 9.1 & $\begin{array}{l}\text { La identificación del cliente con la imagen de las entidades } \\
\text { financieras o bancarias (sentido de pertenencia) influye en } \\
\text { la utilización de los medios móviles. }\end{array}$ & & & & \\
\hline 9.2 & $\begin{array}{l}\text { La imagen de una entidad bancaria le garantiza respaldo en } \\
\text { caso que se presente un caso fortuito a la hora de realizar } \\
\text { sus operaciones por medio de pagos móviles. }\end{array}$ & & & & \\
\hline
\end{tabular}


María C. Bermeo-Giraldo, Alejandro Valencia-Arias, Bianeth Duque García, Luis F. Garcés-Giraldo y Teresa Luna-Ramírez

\begin{tabular}{|c|c|c|c|c|c|c|}
\hline \multicolumn{2}{|r|}{ Afirmaciones } & 5 & 4 & 3 & 2 & 1 \\
\hline 9.3 & $\begin{array}{l}\text { La reputación de una entidad bancaria o financiera puede } \\
\text { ser relevante en la utilización de los productos ofrecidos, } \\
\text { especialmente si se trata de canales virtuales y móviles. }\end{array}$ & & & & & \\
\hline 9.4 & $\begin{array}{l}\text { Es mejor recurrir a familiares y amigos que a entidades ban- } \\
\text { carias para recibir información sobre los servicios móviles. }\end{array}$ & & & & & \\
\hline 9.5 & $\begin{array}{l}\text { Que alguien cercano a usted use medios de pago móviles } \\
\text { y se los recomiende, influye en su decisión de utilizarlos. }\end{array}$ & & & & & \\
\hline 9.6 & $\begin{array}{l}\text { Para usted es importante conocer la experiencia de alguien } \\
\text { que ya haya utilizado los servicios de pagos móviles para } \\
\text { poder utilizarlo. }\end{array}$ & & & & & \\
\hline 9.7 & $\begin{array}{l}\text { Por desconocimiento sobre el servicio de pagos móviles, us- } \\
\text { ted desaprovecha muchas oportunidades para realizar sus } \\
\text { transacciones personales de forma ágil y rápida. }\end{array}$ & & & & & \\
\hline 9.8 & $\begin{array}{l}\text { Tener conocimiento en temas financieros es importante } \\
\text { para utilizar con más frecuencia medios de pagos móviles. }\end{array}$ & & & & & \\
\hline 9.9 & $\begin{array}{l}\text { El desconocimiento en el uso de plataformas de pago con- } \\
\text { lleva estar en riesgo de fraude, robos o pérdida de infor- } \\
\text { mación confidencial. }\end{array}$ & & & & & \\
\hline 9.10 & $\begin{array}{l}\text { Conocer las entidades que vigilan y regulan estos servicios } \\
\text { de pagos móviles le genera seguridad. }\end{array}$ & & & & & \\
\hline 9.11 & $\begin{array}{l}\text { Las diferentes técnicas de verificación de acceso (proto } \\
\text { colos) como la utilización de usuario y contraseña, le dan } \\
\text { seguridad a la hora de adoptar una aplicación para pagos } \\
\text { móviles de alguna entidad bancaria. }\end{array}$ & & & & & \\
\hline 9.12 & $\begin{array}{l}\text { Si los medios de pagos móviles contaran con mayor con- } \\
\text { trol y vigilancia por entes gubernamentales, más personas } \\
\text { decidirían usarlos. }\end{array}$ & & & & & \\
\hline 9.13 & $\begin{array}{l}\text { La información ofrecida por las aplicaciones y plataformas } \\
\text { de pagos le generan confianza en la utilización de los telé- } \\
\text { fonos móviles para realizar operaciones. }\end{array}$ & & & & & \\
\hline 9.14 & $\begin{array}{l}\text { A la hora de realizar sus pagos móviles, genera más con- } \\
\text { fianza una entidad bancaria por su trayectoria y buena ima- } \\
\text { gen, que una cooperativa. }\end{array}$ & & & & & \\
\hline 9.15 & $\begin{array}{l}\text { La experiencia y opinión favorable de alguien cercano a } \\
\text { usted sobre una entidad bancaria afecta su percepción de } \\
\text { confianza en los dispositivos móviles para realizar transac- } \\
\text { ciones personales. }\end{array}$ & & & & & \\
\hline 9.16 & $\begin{array}{l}\text { Los pagos mediante dispositivos móviles presentan nuevas } \\
\text { oportunidades y nuevos riesgos en su utilización. }\end{array}$ & & & & & \\
\hline
\end{tabular}

